\section{Algunos aspectos éticos sobre el riesgo preconcepcional}

\section{Some ethical aspects of the preconception risk}

Goliat Reina Gómez

Hospital Ginecobstétrico Ramón González Coro. Calle 21, n ${ }^{8}$ 856, entre Calle 4 y Calle 6. Municipio Plaza de la Revolución. Ciudad de La Habana, Cuba. Código Postal 10400.

\begin{abstract}
Quality care in reproductive health services corresponds to an interrelation process involving clients, health dispensers, the health and social systems. The equity index of health encompasses accessibility to health services and prenatal care, maternal and perinatal mortality. Therefore it is necessary to establish ethical principles of Medicine based on more scientific diagnosis of women's health, with absolute respect to reproductive rights that should be placed at the same level of principles regarding individual dignity, equity, non-discrimination, participation and solidarity. It is equally important to address women's health within a collective health vision related to social phenomena. The practice of family and social Medicine cannot be detached from individual practice and doctors must continue to advocate that patients be increasingly and equitably benefited.
\end{abstract}

Key words Reproductive medicine, Ethics, medical, Women's health, Equity in health condition
Resumen La calidad de la atención en los servicios de salud reproductiva responde a un proceso de interrelación entre los usuarios, los prestadores de servicios, el sistema de salud y el sistema social. Los índices de equidad en salud incluyen accesibilidad a los servicios de salud, atención prenatal y mortalidad perinatal y materna y es necesario establecer los principios éticos de la profesión médica sobre la base de diagnósticos más científicos de la salud femenina, con absoluto respeto a los derechos reproductivos, que resultan equiparables a los principios de dignidad individual, equidad y no-discriminación, participación y solidaridad, resultando importante establecer el abordaje de la salud femenina como visión de salud colectiva dentro de los fenómenos sociales. La medicina familiar y social no puede separarse de la práctica individual y los médicos deben continuar abogando porque los pacientes obtengan los mayores beneficios en forma equitativa.

Palavras-chave Medicina reproductiva, Etica medica, Salud de las mujeres, Eqüidad en el estado de salud 
Uno de los aspectos cuya demanda aumentará previsiblemente en la atención primaria de salud es la atención en salud reproductiva. Teniendo en cuenta que el conocimiento médico basado en la evidencia, los criterios consensuados entre médicos de atención primaria y especialistas y las guías de buena práctica clínica definirán las actitudes, valores y expectativas en la atención primaria y que la información modificará las relaciones con los pacientes, al aumentar el nivel de conocimientos de la población, 1 se hace necesario prestar atención a los principios éticos profesionales.

Desde el punto de vista técnico-científico, resulta interesante señalar que en el siglo XIX Jaques Von Siebold citado por O' Dowd e Philipp (1998: 3)2 planteaba que el origen de la Obstetricia se encontraba en el tratamiento de situaciones normales, lo que le diferenciaba de la Medicina. Esto significa que se consideraban los riesgos como parte del proceso natural de reproducción, enfoque que se modifican progresivamente durante los siglos XIX y XX, provocando cambios éticos en el ejercicio de la profesión médica.

En este artículo pretendemos analizar los principios éticos de la profesión médica sobre la base de diagnósticos más científicos de la salud femenina, con absoluto respeto a los derechos reproductivos, que resultan equiparables a los principios de dignidad individual, equidad y no-discriminación, participación y solidaridad. ${ }^{3}$

\section{Desarrollo}

El International Bioethics Committee of United Nations Educational, Scientific and Cultural Organization (UNESCO) ${ }^{4}$ definió la salud reproductiva como "estado de bienestar físico, mental y social", que reconoce las diferencias entre los sexos, con aproximación interdisciplinaria e intersectorial, reflejando un enfoque sociocultural, físico y psicológico.

La calidad de la atención en los servicios de salud reproductiva responde a una estructura básica de servicios (recursos, organización, disponibilidad y prestación de servicios) en un proceso continuo de interrelación entre los usuarios, los prestadores de servicios, el sistema de salud y el sistema social. Este proceso puede verse afectado por prácticas inadecuadas o falta de capacitación, no sólo en aspectos técnicos, sino en aquellos que involucran la comunicación con los usuarios. 5

Los principios éticos implican obligaciones concretas por parte de los organismos internacionales, gobiernos, dispensadores de salud y sociedad en su conjunto, y también atañe a los profe- sionales, personas y grupos de población. ${ }^{6}$

El conocimiento de los riesgos a que se ve expuesta la mujer durante la gestación, el parto y el puerperio es tan viejo como la propia humanidad, pero solo el desarrollo de la Higiene y su vinculación con los procesos sociales a partir de los siglos XVIII y XIX, al promover la investigación estadística, crea las condiciones para identificar los factores de riesgo. Es este vínculo original lo que nos obliga a incluir en un análisis ético la situación sociopolítica y económica de los últimos años, que ha incrementado la desigualdad y la inequidad de la atención médica en el llamado Tercer Mundo, sirviendo como ejemplo el deterioro de la calidad de la atención en América Latina y el Caribe, 7 unidad histórica y geográfica que nos incluye, donde los resultados son relativamente pobres en función del volumen de recursos que se le destinan, siendo necesario evaluar la eficiencia técnica y asignativa de cantidades cada vez más decrecientes y enfatizar la función del Estado como garantía del acceso a los servicios sociales. ${ }^{8}$ No es casual que al establecer índices de equidad en salud se incluyan la accesibilidad a los servicios de salud, la atención prenatal y la mortalidad perinatal y materna. La lucha por la equidad no debe partir de sentimientos de compasión o espíritu de justicia, sino del análisis objetivo de que es producto del sojuzgamiento de una parte de la humanidad por la outra. 9

Recordemos que el enfoque de riesgo es un método de trabajo basado en la observación de que la probabilidad de ocurrencia para un evento es mayor en determinado grupo de personas, familias o comunidades (todas las mujeres embarazadas corren el riesgo de sufrir complicaciones, aunque algunas estén más expuestas que otras). Esto supone cuidados no igualitarios y requiere que los recursos se distribuyan en función de dar más a quienes tengan mayor necesidad. 10 Se necesita para ello una adecuada identificación de los riesgos, información sobre los mismos, discusión de su repercusión sobre la gestación desde la perspectiva materno-fetal, referencias al consejo genético, aspectos psicológicos y sociales de la preparación previa del embarazo, posibilidad de espaciamiento entre los mismos y planeamiento de los cuidados prenatales, todo lo cual debe conducir a la introducción de intervenciones apropiadas y deseadas.

La evaluación preconcepcional debe intentar prevenir las condiciones en que se desarrolla un embarazo, tratando de minimizar los efectos negativos, ya que la complejidad de los sistemas biológicos, capaces de mantener estructuras ordenadas en condiciones alejadas del equilibrio, ocasiona dificultades 
para establecer predicciones, por lo que es necesario una ética responsable, capaz de prever consecuencias remotas, que incorpore el respeto y la esperanza sin aceptar el quietismo nosológico.11

Los elementos de riesgo están presentes en ambos sexos, pero repercuten fundamentalmente en la mujer. El primer problema es el enfoque paternalista tradicional que han tenido los problemas de salud femeninos, que llegaron a causar perjuicio a la mujer, al hacerle sentir y creer que algunos de sus síntomas no eran reales o resultaban intratables, como condición inherente a su sexo y las escasa asignaciones de recursos para los programas de salud dirigidos a la mujer. ${ }^{12}$ La estructura jerárquica en que el médico domina el sistema, desde un enfoque tradicional de roles, y las políticas y prioridades basadas en la perspectiva reproductiva, reflejo de valores personales, religiosos o culturales de médicos, familiares y sociedad, implica en ocasiones un reto a la autonomía de la mujer y a su salud mental y psicosocial. ${ }^{13}$ Más que como objeto sexual, la mujer se ha visto como sujeto reproductivo. 14

Las diferencias en el abordaje de la salud femenina como visión de Salud Pública o Salud Colectiva están en el que hacer colectivo de los fenómenos sociales. ${ }^{15}$ La mujer, al preservar y transmitir tradiciones y costumbres, logra percibir necesidades y hallar medios de solucionarlas y sus necesidades estratégicas sólo pueden ser satisfechas a largo plazo, como parte integral del desarrollo humano sostenido, y su posible solución está en la creación de conciencia y autoestima a través de la educación y la movilización política.

En la década del 70 la profesión médica se vio tan implicada en el cuidado de la salud femenina por la invasión de la privacidad con la tecnología que se hizo necesario dar mayor participación a las pacientes en la toma de decisiones. ${ }^{2}$ Más recientemente, la Federación Internacional de Ginecologia y Obstetricia (FIGO) ha establecido los aspectos éticos de la reproducción humana y salud de la mujer 16 basándose en los acuerdos establecidos por la Conferencia de Beijing, que ratificaban los principios postulados en la reunión de El Cairo.

La obtención del consentimiento informado para la realización de procedimientos como la utilización de anticonceptivos17,18 o la esterilización quirúrgica, 19,20 resulta requisito indispensable para la buena práctica médica y es posible que ello se extienda en un futuro a la utilización del ultrasonido diagnóstico, una vez concluido el estudio iniciado en octubre de 1997 por la Organización Mundial de la Salud (OMS), o al uso de medicamentos para el tratamiento de la infertilidad o durante el embarazo, por los riesgos que de ellos se derivan el consentimiento informado no es un simple documento formal, sino debe ser el resultado de la educación del paciente, 21 a partir de los conocimientos médicos sobre factores biológicos (susceptibilidad, exposición, inmunidad), psicológicos (acontecimientos de vida, tensiones, creencias de salud y estilos de vida) 22 y las diferencias culturales, 23 que pueden constituir elementos de enfrentamiento entre médicos y pacientes. No resulta gratuita la acusación hecha a los servicios de salud reproductiva de estar exclusivamente implicados en el control de la fertilidad, lo que refleja fallos en la comunicación de la información, abandono de la sensibilidad y trato deshumanizado.

Según Parker y Hope, 24 el mayor efecto que la ética médica ha sufrido es en el campo de la información a los pacientes, que ha tomado el modelo del consumidor ideal (bien informado y libre de hacer su elección) para construir el proceder médico ideal. La información tecnológica aportará cambios sociales en la esfera de las determinantes biológicas del desarrollo humano, pero el mayor impacto será el incremento de la globalización, que pondrá de manifiesto que el principal problema ético en Medicina lo constituye la falta de un adecuado sistema de salud ventajoso para la mayor parte del mundo; se hace necesario un mayor interés por la distribución de recursos y beneficios a los más pobres y por la protección lingüística y cultural de los pueblos. ${ }^{24}$

Los objetivos expresados por la Asociación de Escuelas de Medicina de América incluyen el compromiso de defender en todo momento los intereses de los pacientes, exigiendo el compromiso de proveer asistencia los que se encuentren imposibilitados de pagar y refuerza la necesidad del conocimiento médico de los factores económicos, sociales y culturales que contribuyan a la salud. 25 Resulta también importante tener estudiantes que aprendan de médicos con un rol eficiente en la atención compasiva de los pacientes, especialmente los pobres o médicamente no asegurados. 26

La investigación para una mejor salud reproductiva tiene por objeto ampliar sus opciones, movilizar la gran diversidad de investigación biomédica, social y del comportamiento respecto a la salud reproductiva y la sexualidad y promover la utilización de los resultados para mejorar la formulación y ejecución de las políticas, aumentando su calidad, eficiencia y sensibilidad ante las necesidades de los destinatarios, mejorando la calidad de vida y satisfaciendo necesidades de grupos insuficientemente atendidos y también debe velar por la seguridad y calidad de los métodos de regulación de la fecundidad. $27 \mathrm{La}$ exclusión de la mujer de investigaciones por riesgo de 
embarazo y por los cambios cíclicos hormonales constituye un problema ético y metodológico.

En el aspecto de la responsabilidad individual, es importante recordar que la negligencia médica recoge dos aspectos: de una parte, la utilización de técnicas y conocimientos que han quedado anticuados (negligencia por falta de información continua) y por otra, inobservancia de medidas de seguridad (negligencia en relación con normas en vigor). ${ }^{28}$ Las necesidades de la atención primaria en salud reproductiva han llegado a forzar modificaciones en los programas de estudio de la especialidad. 29
Para el médico de atención primaria, el cuidado individual exige una visión integral que relacione actividades de prevención, promoción, curación y rehabilitación que no podrá descuidarse cuando se analice la función familiar y la atención comunitaria. ${ }^{30}$ La contradicción entre lo que resulta mejor para el individuo y lo que es bueno para la sociedad puede constituir un problema ético. La medicina familiar y social no puede separarse de la práctica individual y los médicos deben continuar abogando porque las pacientes obtengan los mayores beneficios en forma equitativa. 31

\section{Referencias}

1. Artells J, Andrés J. Perfil, actitudes, valores y expectativas de los profesionales de atención primaria en el siglo XXI. Análisis prospectivo Delphi. Cuad Gestión Profes Atención Primaria 1999; 5 Supl 1: 1-123.

2. O' Dowd ME, Philipp EE. Narrativa panoramica actual. In: O'Dowd ME, Philipp EE. Historia de la ginecología y obstetricia. Barcelona: Edika; 1998. v. 1, p. 1-34.

3. Comisión Independiente sobre Población y Calidad de Vida IEPALA. Elegir el futuro. Madrid: Instituto de Estudios Políticos para America Latina e Africa (IEPALA); 1999. p. 203-36.

4. UNESCO (United Nations Educational, Scientific and Cultura Organization), International Bioethics Committee. Bioethics and woman's right ... In: Proceedings of the 5th Session; 1998 Dec 2-4; Noodwijk, the Netherlands. Vincennes: Division of the Ethics of Science and Technology of UNESCO; 1999. V. 1, p. 3-18.

5. OPS (Organización Panamericana de la Salud). Calidad de los servicios de la salud sexual y reproductiva. Washington, DC: OPS; 2000.

6. Bankowski Z. Etica y salud. Foro Mundial Salud 1995; 16: 133-43.

7. Toro-Ocampo H. La salud reproductiva en los programas de salud pública. In: Rodríguez O, Santiso R, Calventi V, editores. Libro de texto de FLASOG [Federación Latinoamericana de Sociedades de Obstetricia y Ginecologia]. Caracas: Ateproca; 1996. v. 1, p. 719-41.

8. Molina R, Pinto M, Henderson P, Vieira C. Gasto y financiamiento en salud: situación y tendencias. Rev Panam Salud Pública 2000; 8: 71-83.

9. Fürntrat-Kloep EF. Equidad, justicia social y democracia. La Habana: Latina; 1996. p. 23-38.

10. Sarué E, Díaz AG, Fescina RH, Simini F, Giacomini H, Schwarz R. Factores de riesgo. Montevideo, Uruguay: Centro Latinoamericano de Perinatología y Desarrollo Humano; 1992. (Publicación Científica, 1264).

11. Jonas H. El principio de la responsabilidad. Turin: Einaudi; 1990.
12. Sttoppard M. Trato dispensado a las mujeres. In: Guía médica para la mujer. 6. ed. Madrid: Grijalbo; 1995. p. 348-51.

13. WHO (World Health Organization). Psychosocial and mental health aspect of women's health. Ginebra: WHO; 1993.

14. Henao G. Ideología, obstetricia y sociedad. IATREIA [Medellín, Colombia] 1999; 12: 64-74.

15. Tajer D, Ynoub RC, Castañeda MH, coordenadores. Relatoria de la Oficina de trabajo "Género y Salud Colectiva. In: VII Congreso Latinoamericano de Medicina Social; 1997 Mar 17-21; Buenos Aires, Argentina. Buenos Aires; 1997.

16. FIGO (Federación Internacional de Ginecología y Obstetricia), Comité. Directrices para los aspectos éticos de la reproducción humana y salud de la mujer. Rev Centroam Obstet Ginecol 1998; 8: 44-8.

17. Coggins C, Segal S. Reproductive health. J Reprod Immunol 1998; 41: 3-5.

18. Fisher WA, Fisher JD. Understanding and promoting sexual and reproductive health behavior: theory and method. Rev Ann Sex Res 1998; 9: 39-76.

19. Zighelboi I. Anticoncepción quirúrgica femenina. In: Rodríguez O, Santiso R, Calventi V, editores. Libro de texto de FLASOG [Federación Latinoamericana de Sociedades de Obstetrícia y Ginecologia]. Caracas: Ateproca; 1996. v. 1, p. 705-18

20. Seoane-Rodríguez JA. Aspectos éticos y jurídicos de la esterilización en pacientes con retraso mental. Cuad Bioética 1999; 10: 140-8.

21. Nuñez de Villavicencio F. Bioética: el médico, un educador. In: Acosta J, editor. Bioética desde una perspectiva cubana. La Habana: Centro Félix Varela; 1997. p. 12832.

22. Morales F. Psicología de la salud. Conceptos básicos y proyecciones de trabajo. La Habana: Cietífico-Técnica; 1998. p. 85-112. 
23. Carrillo E, Green AR, Betancourt JR. Cross-cultural primary care. A patient-based approach. Ann Intern Med 1999; 130: 829-934.

24. Parker M, Hope T. Medical ethics in the 21th century. J Intern Med 2000; 247: 1-10.

25. Swick HM, Szenas P, Danoff D, Whitcomb ME. Teaching professionalism in undergraduate medical education. JAMA 1999; 282: 830-2.

26. Cutillo B. La enseñanza del profesionalismo para estudiantes de medicina. JAMA 2000; 283: 197-8.

27. OMS (Organización Mundial de la Salud). Acción para el siglo XXI. Salud y derechos reproductivos para todos. Family care international. New York: OMS; 1994
28. Povl R. Negligencia médica. Foro Mundial Salud 1996; 17: 227-31

29. Bessonnette JM, Gabbe SG, Hammond CB, MacDonald PC, Polan ML, Robeerts JM. Restructuring residency trainning in obstetric and gynecology. Am J Obstet Gynecol 1999; 180 (Pt 1): 516-8.

30. De la Revilla L. La formación del médico de la familia. Rev Atención Primaria 1999; 23 Suppl 1: 53-60.

31. The Tavistock Group. A shared statement of ethical principles for those who shape and give health care. Ann Intern Med 1999; 130: 144-7. 